A. Pramesh Rao, G. Swarup and Gopal-Krishna, eds.

\title{
HI in Active Galactic Nuclei
}

\author{
Z. Yu and D. Jiang \\ Shanghai Astronomical Observatory, 80 Nandan Road, \\ Shanghai 200030, China
}

\begin{abstract}
Recent VLBA and VLA observations indicate the possibility of a circumnuclear disc or torus of HI in detected in absorption in NGC 3894. Strong absorption lines are detected toward both the aproaching and receding jets. Absorption profiles integrated over six regions across the source are shown. At lease two velocity components are present in all lines of sight except toward the southeast. The parameters are obtained from these fits(Peck and Taylor,1998,Ap.J.,502,L23). We use the bending model to explain the four prominent absorption features.
\end{abstract}

\section{Introduction}

Although too underluminous to be formally classified as a compact symmetric object, NGC3894 shares the characteristics that lead to the detection of $\mathrm{HI}$ absorption in this class of objects. The radio source associated with NGC3894 is compact and oriented such that a circumnuclear disk or torus should be observable against the counterject and possibly against the core and jet as well(Pect and Taylor 1998). Strong absorption lines are detected towards both the aproaching and receding jets. Absorption profiles integrated over six regions across the source are shown in Figure 2 of Pect and Taylor(1998). At least two velocity components are present in all lines of sight except toward the southeast lobe. Evindence for additional weak components can be seen in the integrated profiles. Gaussian functions have been fitted to the two strong absorption features. The parameters are obtained from these fits (Peck \& Taylor 1998). They refer to the four prominent absorption features as A1, A2, B, and C. We use the bending model to explain the four prominent absorption features.

\section{Model and Results}

The bending model was made up of concentric rings with radii starting at, and increasing in some steps. Each ring was allowed to have an arbitrary circular angle. The map of the model is shown in Figure 8 of Rogstad et al.(1974).

In the model $r_{1}$ and $r_{2}$ represent the inner and outer radius of the ring, respectively. $\mathrm{r}$ is the radius of the ring, $\mathrm{Z}$ the thickness of the ring, $\theta$ the inclination angle, $\psi$ the position angle and $\mathrm{x}-\mathrm{y}$ is the coodinate system in the 
ring-plane. So

$$
x^{2}+y^{2}=r^{2} \text {. }
$$

The only radio transition of ground-state neutral hydrogen is the 21-cm hyperfine line at $1420.4048 \mathrm{MHz}$. In contrast to the typically highly saturated ultraviolet lines of HI, a Gaussian 21-cm line has an opacity at line center of

$$
\tau=\frac{5.2 \times 10^{-19} \rho}{\Delta V T_{s}}\left[\frac{\left(r_{2}-r_{1}\right)^{2}\left(r^{2}-y^{2}\right)}{r^{2}}+Z^{2}\right]^{1 / 2} .
$$

where the spin temperature $\mathrm{T}_{s}$ is the kinetic temperature in most cases, and $\Delta \mathrm{V}$ is the lines's full width at half-maximum in kilometers per second. $\rho$ is density of the neutral hydrogen. For a rotating ring the velocity relationship is obtained as follows:

$$
\left(V_{c e n}-V_{s}\right)=V_{t}(y / r) \sin \theta \cos \psi-V_{t}(x / r) \sin \theta \sin \psi
$$

where $\mathrm{V}_{\text {cen }}$ is central velocity, $\mathrm{V}_{s}$ is systemic velocity, $\mathrm{V}_{t}$ is tangential velocity at $r$. Using equations (1)-(3) the location coordinate $\mathrm{x}$ and $\mathrm{y}$, and $\mathrm{r}$ have been obtained. We assume $r_{2}-r_{1}=0.1 \mathrm{r}$ and the parameters $\tau, \Delta \mathrm{V}$, and $\mathrm{V}_{c e n}$ are obtained by fitting the observations as shown in the Peck \& Taylor(1998). According to the our model of the ring we take the parameters as follows: $\theta=90^{\circ}$, $\rho=0.57 \mathrm{~cm}^{-3}, \mathrm{~T}_{s}=50 \mathrm{~K}, \mathrm{Z}=55 \mathrm{pc}, \mathrm{V}_{t}=200 \mathrm{kms}^{-1}$. The computational results for $\mathrm{A} 1, \mathrm{~A} 2, \mathrm{~B}$, and $\mathrm{C}$ are shown in the Table 1 .

\section{Table 1. Our Computational Results}

\begin{tabular}{c|c|c|c|c|c}
\hline Absorption Feature & $\mathrm{x}(\mathrm{pc})$ & $\mathrm{y}(\mathrm{pc})$ & $\mathrm{r}(\mathrm{pc})$ & $\Delta \mathrm{r}(\mathrm{pc})$ & $\psi$ (degree) \\
\hline A1 & 4574 & 321 & 4585 & 458.5 & 45 \\
$\mathrm{~A} 2$ & 1349 & 940 & 1679 & 167.9 & 10 \\
$\mathrm{~B}$ & 1066 & 889 & 1388 & 138.8 & 20 \\
C & 755 & 1007 & 1259 & 125.9 & 60 \\
\hline
\end{tabular}

Thus we obtain parameters of the rings which give rise to the observed HI and find that the different rings have different properties.

\section{References}

Peck, A.B. and Taylor,G.B., 1998, ApJ, 502, L23.

Rogstad, D.H. et al., 1974, ApJ, 193, 309. 